\title{
Is surgical antibiotic prophylaxis necessary for pediatric orchiopexy?
}

A. J. Rensing ${ }^{\mathrm{a}, *}$, B. M. Whittam ${ }^{\mathrm{a}, \mathrm{b}}$, K. H. Chan ${ }^{\mathrm{a}, \mathrm{b}}$, M. P. Cain ${ }^{\mathrm{a}}$, A. E. Carroll ${ }^{\mathrm{b}}$, W. E. Bennett Jr. ${ }^{\text {b }}$

${ }^{a}$ Division of Pediatric Urology, Department of Urology, Indiana University School of Medicine, Indianapolis, Indiana, USA

${ }^{\mathrm{b}}$ Center for Pediatric and Adolescent Comparative Effectiveness Research, Department of Pediatrics, Indiana University School of Medicine, Indianapolis, Indiana, USA

*Corresponding author. 705 Riley Hospital Drive, Suite 4230, Division of Pediatric Urology, Indianapolis, IN46202, USA. Tel.: 001-317-948-8556; fax: 001-317-944-7481.

Email address: arensing@iu.edu (Adam J. Rensing) 


\section{Summary}

Introduction: Surgeons frequently use surgical antibiotic prophylaxis (SAP), despite limited evidence to support its efficacy. Potential adverse events associated with antibiotic use include allergic reaction (including anaphylaxis,), Clostridium difficile infection, and selecting for resistant bacteria. Surgical site infections (SSI) are very rare in patients undergoing clean pediatric urologic procedures. Current guidelines are unclear about the efficacy of surgical antibiotic prophylaxis for prevention of SSI in the pediatric population.

Objective: It was hypothesized that children who received SAP prior to orchiopexy would have no reduction in surgical site infection (SSI) risk but an increased risk of antibiotic-associated adverse events.

Methods: A retrospective cohort study was conducted of all males aged between 30 days and 18 years who underwent an orchiopexy (ICD-9 CM 62.5) in an ambulatory or observation setting from 2004-2015 using the Pediatric Health Information System database. Inpatients and those with concomitant procedures were excluded. Chi-squared or Fisher's exact tests were used to determine the association between SAP and allergic reaction (defined as a charge for epinephrine or ICD-9 diagnosis code for allergic reaction on the date of surgery) and any of the following within 30 days: SSI, hospital readmission or any repeat hospital encounter. Mixed effects logistic regression was performed, controlling for age, race, and insurance, and clustering of similar practice patterns by hospital. 
Results: A total of 71,767 patients were included: median age was 4.6 years, $61.4 \%$ were white, and $49.3 \%$ had public insurance; 33.5\% received SAP. Of these participants, 996/71,767 (1.4\%) had a perioperative allergic reaction and $<0.1 \%$ were diagnosed with an SSI. On mixed effects logistic regression, those who received SAP had 1.2 times the odds of a perioperative allergic reaction compared with those who did not receive SAP $(P=0.005)$. Surgical antibiotic prophylaxis was not associated with decreased rates of SSI, lower hospital readmission, nor a lower chance of a repeat encounter within 30 days.

Conclusions: In patients undergoing orchiopexy, it was found that SAP did not reduce the risk of postoperative SSI, readmissions, or hospital visits. Patients who received SAP had significantly increased odds of perioperative allergic reaction. This demonstrated that the risks of SAP outweigh the benefits in children undergoing orchiopexy.

Keywords: Surgical site infection; Orchiopexy; Surgical antibiotic prophylaxis 


\section{Introduction}

Surgical antibiotic prophylaxis (SAP) refers to the use of perioperative antimicrobial agents to prevent surgical site infections (SSI). In adult urologic surgery, there is universal support for this practice for clean contaminated procedures, contaminated procedures, and dirty procedures. American Urological Association (AUA) guidelines recommend the routine use of SAP only in clean procedures where associated risk factors are present. European guidelines do not routinely recommend their use for clean procedures [1-3]. The variation in appropriate use of SAP in both pediatric and adult populations highlights the ambiguity of this topic [4-9]. Decisions about SAP use in the pediatric population are complicated by the lack of guidelines.

Several animal and human studies have demonstrated the benefits of perioperative antibiotic prophylaxis [10-13]. However, a recent study of pediatric urology patients demonstrated a very low rate of postoperative infection $(0.8 \%)$ across all wound classifications and surgical sites [14]. There was no demonstrable difference in SSI for clean procedures with or without SAP, with most SSI occurring in the clean-contaminated surgeries (which universally received SAP). They also noted no antibiotic-related adverse events. Given the low rates of SSI (especially with clean procedures) and lack of adverse events, the authors suggested focusing upon clean-contaminated procedures for future recommendations and studies [14].

Multiple recent studies of pediatric surgery patients have demonstrated that children who received SAP were at a significantly increased risk of receiving epinephrine (a surrogate for allergic reaction) and Clostridium difficile (C. difficile) infection compared with those who did not receive SAP $[4,15]$. Macy et al. demonstrated in a retrospective analysis that cephalosporins 
led to an increased risk of $C$. difficile infection, anaphylaxis, nephropathy $(0.15 \%)$ and all-cause mortality within 1 day $(0.10 \%)$ [16]. Wang et al. linked acetaminophen and/or antibiotic exposure within the first year of life with certain lifelong diseases, including atopic dermatitis, asthma, and allergic rhinitis [17]. Multiple studies have documented cephalosporin crossreactivity with other $\beta$-lactam antibiotics, and the $\beta$-lactam family is the most common cause of hypersensitivity drug reactions $[18,19]$. From a population health standpoint, the inappropriate use of antibiotics inevitably results in the selection of resistant organisms in the community, leading to future morbidity and healthcare costs, especially with respect to methicillin-resistant Staphylococcus aureus infections in infants [20-23]. A previous study showed that SAP given during pediatric circumcision had no association with SSI, penile reoperation, or hospital visit on bivariate analysis, but did lead to an increased risk of allergic reaction or hospital visit on multivariate analysis [24].

The current study sought to evaluate, using the Pediatric Health Information System (PHIS) database, the effect of SAP at the time of orchiopexy on rates of SSI, readmission, reoperation, and antibiotic-associated adverse events. It was hypothesized that children who received SAP prior to outpatient orchiopexy would have no reduction in SSI risk, but an increased risk of antibiotic-associated adverse events (AAAE). 


\section{Materials and Methods}

\section{Data source}

The PHIS is an administrative database that contains inpatient, emergency department, ambulatory surgery and observation encounter-level data from over 45 not-for-profit, tertiary care pediatric hospitals in the United States. These hospitals are affiliated with the Children's Hospital Association (Overland Park, KS). Data quality and reliability are assured through a joint effort between the Children's Hospital Association and participating hospitals. Portions of the data submission and data quality processes for the PHIS database are managed by Truven Health Analytics (Ann Arbor, MI). For the purposes of external benchmarking, participating hospitals provide discharge/encounter data, including demographics, diagnoses, and procedures. The majority of these hospitals also submit resource utilization data (e.g. pharmaceuticals, imaging, and laboratory) into PHIS. Data are de-identified at the time of data submission, and subjected to a number of reliability and validity checks before being included in the database. The present study included data from 43 children's hospitals.

\section{Study population}

A retrospective cohort study was performed of all males aged between 30 days and 18 years who underwent an orchiopexy (ICD-9-CM 62.5) with or without herniorraphy (ICD-9-CM 53.0-53.1) in an ambulatory or observation setting from January 2004 to December 2015. Patients admitted for observation were included to avoid missing patients whose status changed from ambulatory to observation due to an intra-operative adverse event such as a perioperative allergic reaction 
(PAR). Of note, a patient was categorized as having received SAP if a charge was found on the day of surgery for an antibiotic. It was assumed that patients with SAP charges received the medication. The authors felt confident in the validity of the PHIS pharmacy charges, based on the validation study by Chan et al. [25]. Inpatients and those who had length of stay $>2$ days were excluded, as were cases with concurrent procedures, to ensure that the use of SAP was associated with orchiopexy alone (Fig. 1). The cohorts that did and did not receive SAP were then compared.

\section{Calculation of antibiotic-associated adverse event rates}

To characterize the incidence of PAR associated with the administration of SAP, the algorithm proposed by Macy et al. was used [26]. All patients with a charge for epinephrine (as a surrogate event for an allergic reaction) or an ICD-9 diagnosis code for an allergic reaction on the date of surgery were identified (see Appendix 1- ICD9 for allergic reaction). ICD-9 diagnosis codes were included for specific types of drug reactions (e.g. dermatitis) as well as drug reactions where the specific type of reaction was unknown or not documented.

Thirty-day postoperative antibiotic-associated adverse events were classified as follows: 1) perioperative allergic reaction, 2) re-operation, 3) infection within 30 days, 4) day repeat encounter for any reason, 5) allergic reaction within 30 days, and 6) hospital admission. Reoperation was defined as any testicular surgery within 30 days of the orchiopexy (Appendix 2). Surgical site infections were defined as any emergency department visit or hospital readmission that included either of the following ICD-9 diagnosis codes: 998.51 (infected postoperative seroma) or 998.59 (other postoperative infection). 
Statistical analysis

Bivariate analyses (Chi-squared and Fisher's exact test) were performed to determine the association between SAP and the four binary perioperative outcome variables:

1. Perioperative allergic reaction

2. Re-operation

3. 30-day SSI rate

4. 30-day repeat hospital encounter.

Mixed effects logistic regression was then performed, including a random effect for hospital to control for clustering of similar practice patterns within institutions. Categorical age, race, ethnicity, surgeon type (pediatric surgeon or pediatric urologist), and type of insurance were modeled as fixed effects. Each of the four outcome variables were used as dependent variables in four different models. The R software package (http://www.r-project.org) and the lme4 library were used for mixed effects models (http://cran.r-project.org/package=lme4/). $P$-values $<0.05$ were considered significant. The Institutional Review Board at Indiana University approved this study.

\section{Results}

A total of 91,919 males who underwent orchiopexy in an ambulatory or observation setting during the study period were identified. Of these, 20,243 who underwent concurrent procedures (other than hernia repair) were excluded, leaving 71,676 patients for analysis. Of these patients, $33.5 \%(23,986)$ were given SAP and $66.5 \%(47,690)$ were not (Fig. 1$)$. 
Patient characteristics are shown in Table 1. The median age and mean age was 3 years and 4.6 years, respectively (interquartile range: 1,8$)$. A total so $49.3 \%(34,896)$ had public insurance, and $61.0 \%(43,152)$ were white. Urologists performed $81.0 \%$ of all orchiopexies and $45.2 \%$ had a concurrent herniorrhaphy. During the procedure (PAR), 1.4\% (996 patients) received epinephrine or diphenhydramine. During the first 30 postoperative days, 21 soft tissue infections (SSI) were identified $(<0.1 \%)$.

On mixed effects logistic regression, patients who received SAP had a $21 \%$ increased risk of a PAR compared to those who did not receive SAP (OR 1.21, 95\% CI 1.06-1.38, $P=0.005$ ). The SAP group did not significantly differ in the rate of immediate allergic reaction (during encounter) $(P=0.438)$, SSI within 30 days $(P=0.385)$, hospital admission (0.379), or 30-day repeat encounter of any kind $(P=0.065)$ (Table 2$)$.

The study then examined how the relative proportion of patients with orchiopexies who received SAP changed over time (Fig. 2). This rate increased over the course of the study from approximately $20 \%$ to $40 \%$ of all cases (2004-2015), with an $\mathrm{R}^{2}$ value of 0.36549 . Of note, during the study period, hospitals joined the PHIS data set. To control for any inter-hospital variation, a mixed effects model was used. Thus, for the model-based comparisons, there should have not been any confounding hospital variation. 
Lastly, the study sought to access the degree of inter-hospital variability of SAP use for orchiopexy, and this was quite variable, ranging from 3-88\% of all orchiopexy cases performed at an individual hospital (Fig. 3).

\section{Discussion}

Of nearly 71,000 patients undergoing orchiopexy, with or without herniorrhaphy, it was found that SAP did not reduce the risk of postoperative surgical site infection, readmission, or repeat encounter, but it did significantly increase the risk of PAR. Interestingly, when divided into subgroups based upon age, those aged $>1$ year were just as unlikely to have an infection (95\% CI crossed 1) (Table 2).

A previous study demonstrated that SAP provided no benefit in pediatric circumcisions, with a clear increased risk of adverse events [24]. Previous studies have also suggested the safety of eliminating SAP in pediatric hernia repair and orchiopexy; however, relatively small cohorts of patients and rare incidence of postoperative surgical site require a large patient cohort to determine if a true benefit (or detriment) exists [27]. There is also evidence in the adult literature that SAP does not decrease the risk in certain clean procedures, including microsurgical varicocelectomy, adrenalectomy, partial nephrectomy, and nephrectomy [28,29]. Other studies have demonstrated that topical antibiotic prophylaxis may be more beneficial in adult microsurgical varicocelectomies (a clean, adult operation somewhat analogous to pediatric orchiopexy) [30]. 
The current study also discovered an increased risk of an array of adverse events associated with SAP. Immediate allergic reaction was found to have an OR of 1.21 in the group given SAP, as defined by the use of epinephrine or diphenhydramine or use of any of the previously stated ICD-9 diagnosis codes for a drug reaction during the surgical encounter (Appendix 1).

As mentioned in previous studies, a lack of evidence-based guidelines regarding SAP for this common procedure has lead to significant variability in the use of SAP. This finding has been demonstrated with other clean and clean-contaminated procedures in pediatrics, such as circumcision, inguinal hernia repair, hydrocele repair, laparoscopic varicocelectomy, hypospadias repair, and endoscopic urological procedures, and spinal procedures [5,31]. Of further interest in a study by Chan et al., those using SAP for clean procedures had a higher likelihood of using SAP for clean-contaminated procedures. In contrast, those not using SAP for clean-contaminated procedures, had a higher likelihood of not using SAP for clean procedures either.

The most recent AUA Best Practice Policy Statement recommends SAP for clean-contaminated, adult, urologic procedures, and none for clean procedures, unless there are 'risk factors' [1]. A multi-society guideline also recommends not using SAP for clean pediatric procedures [32]. European guidelines have proposed similar recommendations. To address growing concerns of drug-resistant strains of bacteria, some have called for a renewed focus on antibiotic stewardship, especially in the treatment of children [33,34]. 
The increasing use of perioperative antibiotics can only be speculated (Fig. 2). It is possible that 'minor' surgery has increasingly been performed at outpatient surgery centers. With an interest in efficiency, these centers may lean toward requiring or 'encouraging' perioperative antibiotics for all surgeries/procedures as a 'quality measure'; however, this can only be speculated.

This study had some important strengths. By using the PHIS database, it was able to develop a large cohort of patients. This allowed relatively rare outcomes to be studied (e.g. immediate allergic reactions). By collecting patients throughout the US, and by modeling hospitals as random effects, it was able to minimize bias related to particular centers or local patient populations. It also studied a relatively common surgical procedure that is relatively commonly performed by pediatric urologists (and pediatric surgeons).

There were also notable limitations to this study. The most important were those related to the collection of administrative data. With this data set, the study was reliant upon the veracity of the 43 reporting institutions' billing and diagnosis documentation. To ensure accuracy, the Children's Hospital Association reviews PHIS data for accuracy on a quarterly basis. As with all relatively large data sets, there is a certain limit to the level of granularity. Similarly, the study could not capture data related to postoperative course at geographically local primary care offices or emergency rooms. Also, given the inclusion of only free-standing, non-for-profit, US children's hospitals, those treated at other institutions were not captured. In addition, it was unable to access any outpatient physician records (clinic, telephone, or other) to verify the lack of AEs (SSI or otherwise). Theoretically, it could have underestimated the rate of AEs in both groups. Given the relatively broad definition of an allergic reaction, it could also have 
overestimated the true incidence. Epinephrine and diphenhydramine use could have been for a number of reasons, including allergy to any other agent received during the surgical encounter. Without antigen challenge test results any 'true' allergic reaction cannot be confirmed. However, this method has been utilized in previous studies [16]. Lastly, given that only those treated through a general outpatient setting (no inpatient stays $>2$ days) and without concurrent surgical procedures, the patient population likely excluded a small minority of patients with significant medical morbidities.

\section{Conclusion}

This study found convincing evidence to suggest that SAP for pediatric orchiopexy has no clear benefit in the prevention of SSI. In addition, it demonstrated a statistically significant increased risk of immediate allergic reaction. Given these findings, the current institution has decided to limit antibiotic use for SAP for orchiopexy. Further study in other pediatric urology surgeries is invited to further elucidate the benefits and risks associated with SAP for pediatric patients.

\section{Conflict of Interest/Funding: None.}




\section{References}

1. Wolf JS, Jr., et al. Best practice policy statement on urologic surgery antimicrobial prophylaxis. J Urol 2008;179(4):1379-90.

2. Grabe M. Antibiotic prophylaxis in urological surgery, a European viewpoint. Int J Antimicrob Agents 2011;38 Suppl:58-63.

3. Naber KG, et al. EAU guidelines for the management of urinary and male genital tract infections. Urinary Tract Infection (UTI) Working Group of the Health Care Office (HCO) of the European Association of Urology (EAU). Eur Urol 2001;40(5):576-88.

4. Rangel SJ, et al. Recent trends in the use of antibiotic prophylaxis in pediatric surgery. $\mathbf{J}$ Pediatr Surg 2011;46(2):366-71.

5. Chan KH, et al. Variation in Surgical Antibiotic Prophylaxis for Outpatient Pediatric Urological Procedures at United States Children's Hospitals. J Urol 2017;197(3 Pt 2):944-50.

6. Bratzler DW, et al. Use of antimicrobial prophylaxis for major surgery: baseline results from the National Surgical Infection Prevention Project. Arch Surg 2005;140(2):174-82.

7. Giusti A, et al. Surgical antibiotic prophylaxis in children: a mixed method study on healthcare professionals attitudes. BMC Pediatr 2016;16(1):203.

8. Ciofi degli Atti M, et al. Surgical antibiotic prophylaxis in children: adherence to indication, choice of agent, timing, and duration. Eur J Clin Pharmacol 2015;71(4): 4838.

9. Cek M, et al. Antibiotic prophylaxis in urology departments, 2005-2010. Eur Urol 2013; 63(2):386-94.

10. Burke JF. The effective period of preventive antibiotic action in experimental incisions and dermal lesions. Surgery 1961;50:161-8. 
11. Shapiro M, et al. A decisive period in the antibiotic prophylaxis of cutaneous lesions caused by Bacteroides fragilis in guinea pigs. J Infect Dis 1980;141(4):532.

12. Polk HC, Jr, Lopez-Mayor JF. Postoperative wound infection: a prospective study of determinant factors and prevention. Surgery 1969;66(1):97-103.

13. Stone HH, et al. Antibiotic prophylaxis in gastric, biliary and colonic surgery. Ann Surg 1976;184(4):443-52.

14. Ellett $\mathrm{J}$, et al. Post-surgical infections and perioperative antibiotics usage in pediatric genitourinary procedures. J Pediatr Urol 2015;11(6):358.e1-6.

15. Sandora TJ, et al. National variability and appropriateness of surgical antibiotic prophylaxis in us children's hospitals. JAMA Pediatrics 2016;170(6):570-6.

16. Macy E, Contreras R. Adverse reactions associated with oral and parenteral use of cephalosporins: A retrospective population-based analysis. J Allergy Clin Immunol 2015; 135(3):745-52.e5.

17. Wang J-Y, et al. Acetaminophen and/or antibiotic use in early life and the development of childhood allergic diseases. Int J Epidemiol 2013; 42(4):1087-99.

18. Dona I, et al. Trends in hypersensitivity drug reactions: more drugs, more response patterns, more heterogeneity. J Investig Allergol Clin Immunol 2014; 24(3):143-53; quiz $1 \mathrm{p}$ following 153.

19. Buonomo A, et al. Cross-reactivity and tolerability of cephalosporins in patients with cell-mediated allergy to penicillins. J Investig Allergol Clin Immunol 2014;24(5):331-7.

20. Bryce A, et al. Global prevalence of antibiotic resistance in paediatric urinary tract infections caused by Escherichia coli and association with routine use of antibiotics in primary care: systematic review and meta-analysis. BMJ 2016;352:i939. 
21. Cullen IM, et al. An 11-year analysis of the prevalent uropathogens and the changing pattern of Escherichia coli antibiotic resistance in 38,530 community urinary tract infections, Dublin 1999-2009. Ir J Med Sci 2013;182(1):81-9.

22. Ericson JE, et al. Burden of Invasive Staphylococcus aureus Infections in Hospitalized Infants. JAMA Pediatr 2015; 169(12):1105-11.

23. Iwamoto M, et al. Trends in invasive methicillin-resistant Staphylococcus aureus infections. Pediatrics 2013;132(4):e817-24.

24. Chan $\mathrm{KH}$, et al. Adverse events associated with surgical antibiotic prophylaxis for outpatient circumcisions at US children's hospitals. J Pediatr Urol 2017;13(2):205.e1205.e6.

25. Chan $\mathrm{KH}$, et al. Validation of antibiotic charges in administrative data for outpatient pediatric urologic procedures. J Pediatr Urol 2017;13(2):185-6.

26. Macy E, Contreras R. Adverse reactions associated with oral and parenteral use of cephalosporins: A retrospective population-based analysis. J Allergy Clin Immunol 2015; 135(3):745-52 e5.

27. Vaze D, Samujh R, Narasimha Rao KL. Risk of surgical site infection in paediatric herniotomies without any prophylactic antibiotics: A preliminary experience. Afr J Paediatr Surg 2014;11(2):158-61.

28. Richardson I, Nagler HM. Systemic antibiotic prophylaxis not needed for microsurgical varicocelectomy. Urology 2008;71(4):669-71.

29. Kijima T, et al. Antimicrobial prophylaxis is not necessary in clean category minimally invasive surgery for renal and adrenal tumors: a prospective study of 373 consecutive patients. Urology 2012;80(3):570-5. 
30. O'Connor LT, Jr, Goldstein M. Topical perioperative antibiotic prophylaxis for minor clean inguinal surgery. J Am Coll Surg 2002;194(4):407-10.

31. McLeod LM, et al. Perioperative antibiotic use for spinal surgery procedures in US children's hospitals. Spine (Phila Pa 1976) 2013;38(7):609-16.

32. Bratzler DW, et al. Clinical practice guidelines for antimicrobial prophylaxis in surgery. Surg Infect (Larchmt) 2013;14(1):73-156.

33. Wagenlehner FM, et al. Antibiotic stewardship: a call for action by the urologic community. Eur Urol 2013;64(3):358-60.

34. Nicolini G, Sperotto F, Esposito S. Combating the rise of antibiotic resistance in children. Minerva Pediatr 2014;66(1):31-9. 
Table 1. General demographics.

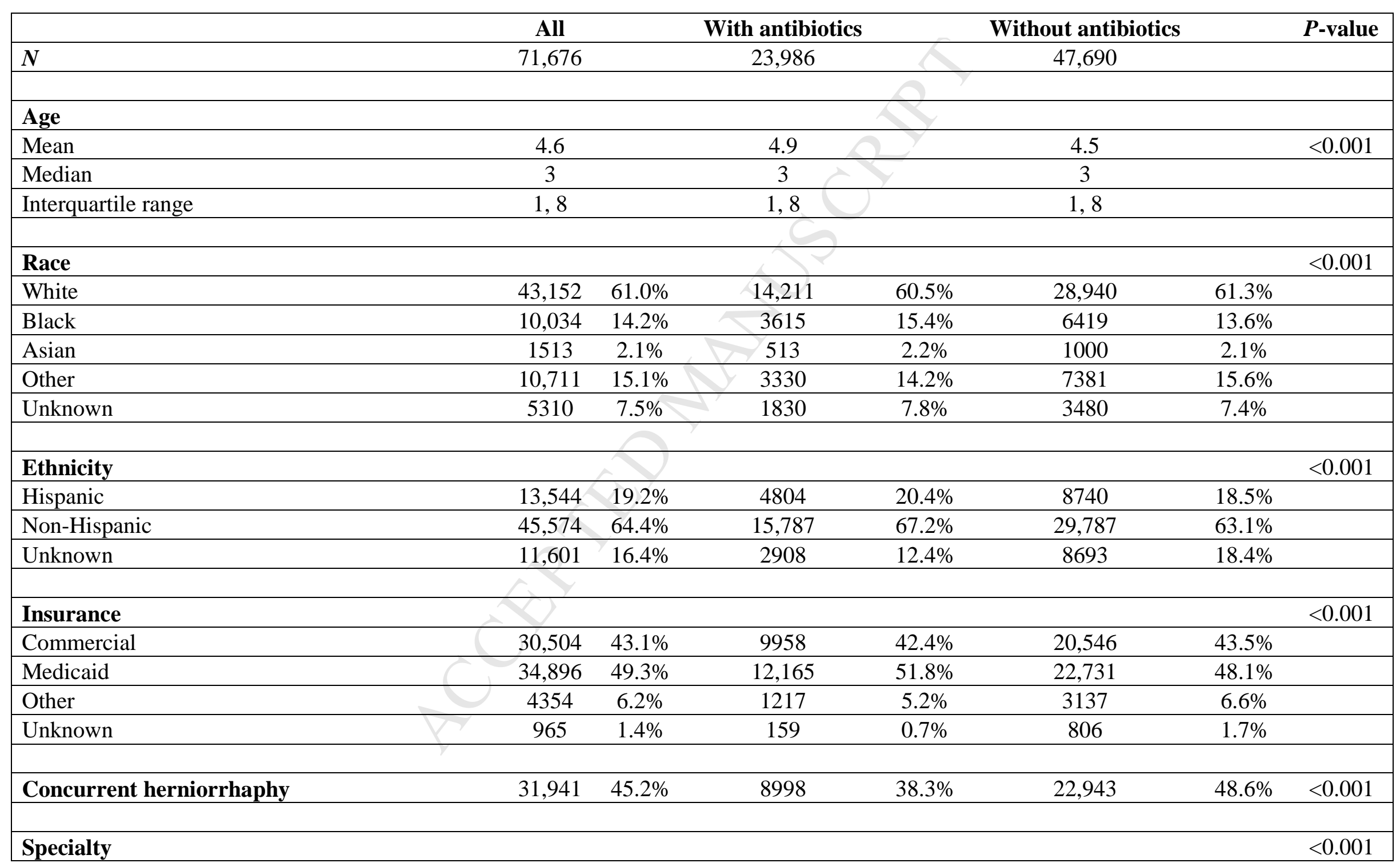




\begin{tabular}{|c|c|c|c|c|c|c|c|}
\hline Urology & 57,304 & $81.0 \%$ & 20,356 & $86.6 \%$ & 36,948 & $78.2 \%$ & \\
\hline Pediatric Surgery & 8431 & $11.9 \%$ & 1866 & $7.9 \%$ & 6565 & $13.9 \%$ & \\
\hline \multirow[t]{2}{*}{ Other/unknown } & 4984 & $7.0 \%$ & 1277 & $5.4 \%$ & 3707 & $7.9 \%$ & \\
\hline & \multicolumn{7}{|c|}{ 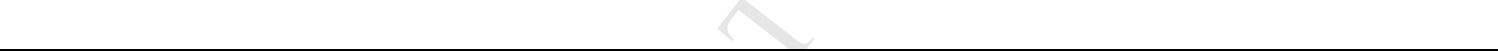 } \\
\hline Allergic reaction & 996 & $1.4 \%$ & 360 & $1.5 \%$ & 636 & $1.3 \%$ & 0.07 \\
\hline \multicolumn{8}{|c|}{ 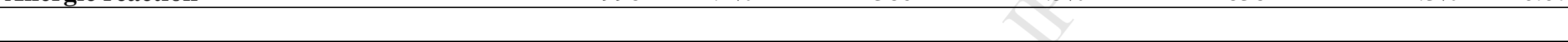 } \\
\hline Soft tissue infection within 30 days & 21 & $<0.1 \%$ & 9 & $<0.1 \%$ & 12 & $<0.1 \%$ & 0.51 \\
\hline Clostridium difficile infection within 30 days & 5 & $<0.1 \%$ & 3 & $<0.1 \%$ & 2 & $<0.1 \%$ & 0.77 \\
\hline
\end{tabular}


Table 2. Pre-operative variables and postoperative outcomes.

\begin{tabular}{|c|c|c|c|c|c|c|c|c|}
\hline & \multicolumn{2}{|c|}{ Perioperative allergic reaction } & \multicolumn{2}{|c|}{30 day SSI } & \multicolumn{2}{|c|}{ Hospital admission } & \multicolumn{2}{|c|}{30 day repeat encounter } \\
\hline & OR $(95 \%$ CI $)$ & $P$-value & OR (95\% CI) & $P$-value & OR $(95 \%$ CI $)$ & $P$-value & OR $(95 \%$ CI $)$ & $P$-value \\
\hline \multicolumn{9}{|l|}{ Age } \\
\hline $1-2$ & $1.04(0.88-1.22)$ & 67578443599307 & $0.39(0.13-1.20)$ & $.10122931550252^{\prime}$ & $0.83(0.68-1.00)$ & $05289437462520 \mathrm{C}$ & $0.94(0.87-1.02)$ & .14830691724844 \\
\hline $3-5$ & $0.73(0.60-0.89)$ & 102230965738198 & $0.13(.020-1.01)$ & .05091681700248 & $0.6(0.47-0.77)$ & $<0.001$ & $0.65(0.59-0.71)$ & $<0.001$ \\
\hline $6-10$ & $0.44(0.36-0.55)$ & $<0.001$ & $0.31(0.08-1.16)$ & 081282539234545 & $0.45(0.35-0.57)$ & $<0.001$ & $0.50(0.46-0.55)$ & $<0.001$ \\
\hline $11-15$ & $0.34(0.25-0.46)$ & $<0.001$ & $0.84(0.25-2.80)$ & .77205741410919 & $0.42(0.30-0.57)$ & $7251693230038 \mathrm{E}$ & $0.59(0.53-0.66)$ & $<0.001$ \\
\hline $16-18$ & & & & & & $\beta>1$ & & \\
\hline \multicolumn{9}{|l|}{ Race } \\
\hline White & - & - & - & - & - & $->$ & - & - \\
\hline Asian & $1.24(0.84-1.83)$ & 28399236061087 & Inf & .99381657620651 & $1.14(0.71-1.83)$ & 1.59622034042736 & $0.90(0.73-1.11)$ & .30575623497794 \\
\hline Black & $1.30(1.09-1.55)$ & 104018374979193 & $0.99(0.26-3.76)$ & .989029627708871 & $1.12(0.90-1.39)$ & .32623950203814 & $1.21(1.11-1.31)$ & $<0.001$ \\
\hline Other & $1.51(1.27-1.81)$ & $<0.001$ & $1.60(0.51-5.02)$ & $41711160836028^{\prime}$ & $0.79(0.63-1.01)$ & .06015083731714 & $1.30(1.20-1.42)$ & $<0.001$ \\
\hline Unknown & $0.37(0.24-0.57)$ & $<0.001$ & $1.11(0.23-5.41)$ & $.90006265783900:$ & $0.76(0.55-1.05)$ & $09811808193773 \mathrm{C}$ & $0.68(0.59-0.78)$ & $<0.001$ \\
\hline & & & & & & & & \\
\hline \multicolumn{9}{|l|}{ Ethnicity } \\
\hline Hispanic & $0.73(0.60-0.88)$ & 101140940002840 & $1.11(0.34-3.64)$ & .86026991488510 & $1.05(0.84-1.31)$ & 65716887865368 & $0.94(0.87-1.03)$ & .17874229957187 \\
\hline Unknown & $0.7(0.57-0.87)$ & 101122892278008 & $1.69(0.55-5.21)$ & $.35967895846429 !$ & $1.19(0.97-1.47)$ & 10137891606191 & $0.67(0.61-0.74)$ & $<0.001$ \\
\hline & & & & & & & & \\
\hline \multicolumn{9}{|l|}{ Insurance } \\
\hline Commercial & - & - & - & 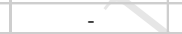 & - & - & - & - \\
\hline Medicaid & $1.24(1.06-1.44)$ & 105905478705892 & $1.77(0.66-4.76)$ & $.25451125670903^{\prime}$ & $1.19(1.00-1.41)$ & 048178020387211 & $1.35(1.27-1.45)$ & $<0.001$ \\
\hline Other & $2.41(1.94-2.99)$ & $<0.001$ & Inf & 98961696765009 & $1.28(0.94-1.74)$ & 12128590779409 & $1.20(1.06-1.36)$ & 105247005831100 \\
\hline Unknown & $1.6(1.07-2.38)$ & 02106985074500 & Inf & .99518954298097 & $1.00(0.51-1.94)$ & .98898459673034 & $0.56(0.40-0.79)$ & 101070413072632 \\
\hline & & & & $12 y=$ & & & & \\
\hline \multicolumn{9}{|l|}{ Service } \\
\hline Urology & - & - & - & - & - & - & - & - \\
\hline Pediatric Surgery & $1.07(0.88-1.31)$ & 48469074223334 & $1.20(0.35-4.14)$ & $.77614584470233^{\prime}$ & $1.76(1.45-2.13)$ & $<0.001$ & $1.67(1.54-1.80)$ & $<0.001$ \\
\hline Other & $2.53(2.11-3.04)$ & $<0.001$ & $0.76(0.10-5.81)$ & .79131328915027 & $1.41(1.06-1.87)$ & $01782730568915 t$ & $1.15(1.02-1.29)$ & 01894590757888 \\
\hline Antibiotics & $1.21(1.06-1.38)$ & 105062334527315 & $1.47(0.61-3.54)$ & $.38530584332725 !$ & $1.07(0.92-1.26)$ & .37925492237794 & $0.94(0.89-1.00)$ & $06513407841059 \varepsilon$ \\
\hline
\end{tabular}


Fig. 1. Orchiopexy cohort.

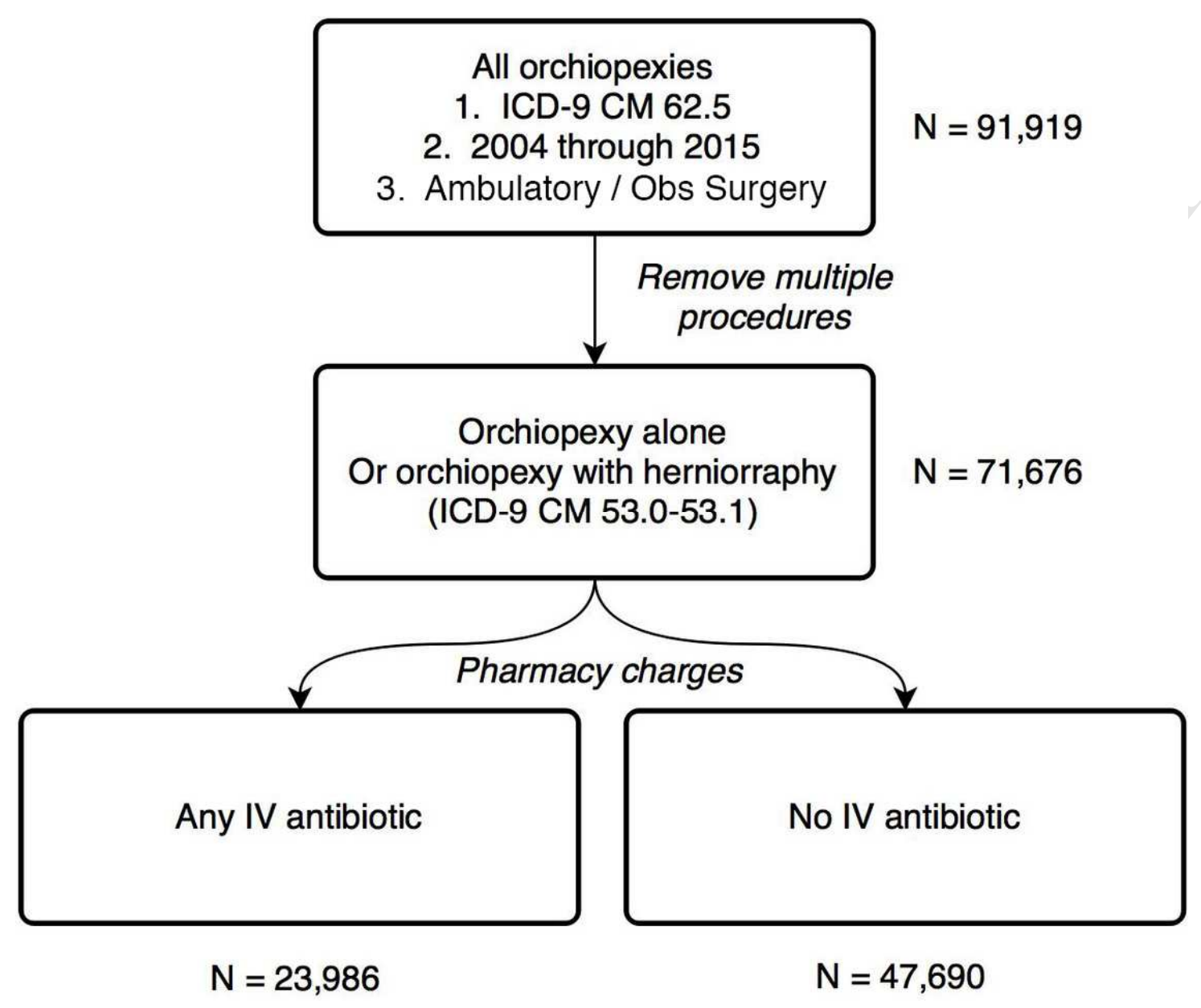


Fig. 2. Antibiotic prophylaxis use variation by year.

Proportion of Orchiopexies Receiving Intraoperative Antibiotics

$50.0 \%$

20.0\%

$0.0 \%$

\begin{tabular}{l|l|l|l|l|l|l|l|l}
2007 & 2008 & 2009 & 2010 & 2011 & 2012 & 2013 & 2014 & 2015
\end{tabular} 
Fig. 3. Antibiotic prophylaxis use variation by hospital.

\% Orchiopexy with IV Antibiotics, Variation by Hospital

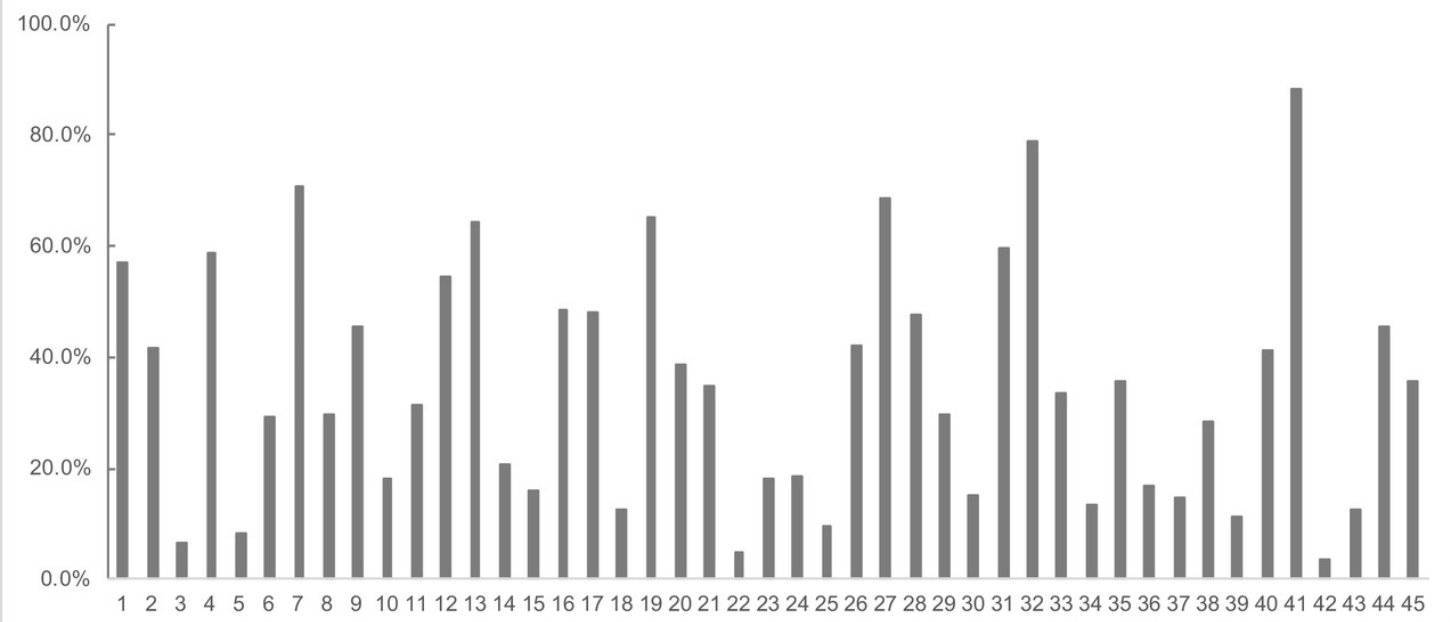


Fig. 4. Antibiotic use variation stratified by hospital size.

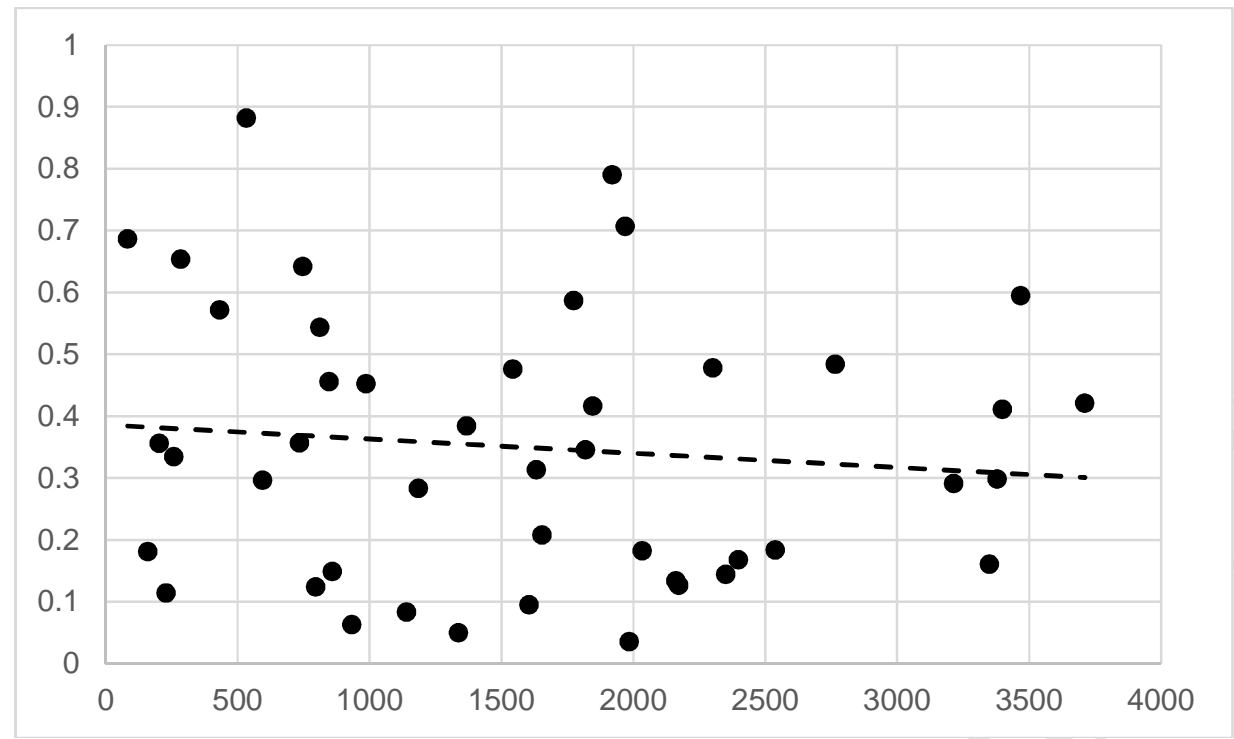

